\title{
Making Personalized Health Care Even More Person- alized: Insights From Activities of the IOM Genomics Roundtable
}

Sean P. David, MD, SM, DPbil ${ }^{1,2}$

Samuel G. Jobnson, PharmD $D^{2-4}$

Adam C. Berger, PbD ${ }^{3}$

W. Gregory Feero, $M D, P b D^{3,5}$

Sharon F. Terry, $M A^{2,6}$

Larry A. Green, $M D^{7}$

Robert L. Pbillips Jr, MD, MSPH

Geoffrey S. Ginsburg, $M D^{2,9}$

'Department of Medicine, Stanford University School of Medicine, Palo Alto, California

${ }^{2}$ Roundtable on Translating Genomic-Based Research for Health, Institute of Medicine (IOM) of the National Academies

${ }^{3}$ Applied Pharmacogenomics, Kaiser-Permanente Colorado, Aurora, Colorado

${ }^{4}$ Department of Clinical Pharmacy, University of Colorado, Denver, Colorado

${ }^{5}$ Maine-Dartmouth Family Medicine Residency Program, Augusta, Maine

${ }^{6}$ Genetic Alliance, Washington, DC

${ }^{7}$ Department of Family Medicine, University of Colorado, Denver, Colorado

${ }^{8}$ American Board of Family Medicine, Lexington, Kentucky

${ }^{9}$ Center for Applied Genomics and Precision Medicine, Duke University School of Medicine, Durham, North Carolina

Conflicts of interest: David is a scientific advisory with BaseHealth, Inc. The authors declare no other conflicts of interest.

\section{CORRESPONDING AUTHOR}

Sean P. David, MD, DPhil

Stanford University School of Medicine Population Health Sciences Building,

Suite 200

1070 Arastradero Rd

Palo Alto, CA 94304

spdavid@stanford.edu

\begin{abstract}
Genomic research has generated much new knowledge into mechanisms of human disease, with the potential to catalyze novel drug discovery and development, prenatal and neonatal screening, clinical pharmacogenomics, more sensitive risk prediction, and enhanced diagnostics. Genomic medicine, however, has been limited by critical evidence gaps, especially those related to clinical utility and applicability to diverse populations. Genomic medicine may have the greatest impact on health care if it is integrated into primary care, where most health care is received and where evidence supports the value of personalized medicine grounded in continuous healing relationships. Redesigned primary care is the most relevant setting for clinically useful genomic medicine research. Taking insights gained from the activities of the Institute of Medicine (IOM) Roundtable on Translating Genomic-Based Research for Health, we apply lessons learned from the patient-centered medical home national experience to implement genomic medicine in a patient-centered, learning health care system.
\end{abstract}

Ann Fam Med 2015;13:373-380. doi: 10.1370/afm.1772.

\section{INTRODUCTION}

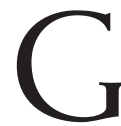
enomic medicine has the potential to transform health care delivery and improve the precision of disease risk prediction, diagnostics, and therapeutics for patients. Personalized medicine has its roots in primary care, where evidence supports the value of continuous healing relationships and a thorough knowledge of individual patients. ${ }^{1}$ Genomic medicine may have its greatest impact when systematically integrated into the primary care setting, where most health care occurs in primary care is delivered ${ }^{2,3}$ personalized through continuous, healing relationships. Francis Collins, director of the National Institutes of Health, has described personalized medicine as a process that will "depend on continued accurate identification of genetic and environmental risk factors, and the ability to utilize this information in the real world to influence health behaviors and achieve better outcomes." ${ }^{\prime \prime 4}$ By applying insights from workshops convened by the Institute of Medicine (IOM) Roundtable on Translating Genomic-Based Research for Health, we propose a model of clinical implementation science to address evidence gaps and guide the integration of genomic medicine into the patient-centered medical home (PCMH).

\section{Realizing the Potential of Genomic Medicine in Primary Care}

Primary care has been defined by the IOM as "the provision of integrated, accessible health care services by clinicians who are accountable for addressing most personal health care needs, developing a sustained partnership with patients, and practicing in the context of family and community." ${ }^{5}$ The key features of primary care (patient-centered, comprehen- 


\section{Table 1. The Definition and Key Features of Primary Care}

\section{Institute of Medicine Definition}

"Primary care is the provision of integrated, accessible health care services by clinicians who are accountable for addressing a large majority of personal health care needs, developing a sustained partnership with patients, and practicing in the context of family and community."

4 Key features of primary care ${ }^{6,7}$

1. It is person rather than disease focused. This focus entails sustained relationships between patients and providers in primary care practices over time, often referred to as continuity.

2. It provides a point of first contact for whatever people might consider a health or health care problem. In properly organized health care systems, primary care ensures access to needed services.

3. It is comprehensive. By definition, it can encompass any problem. Many problems in primary care are ambiguous and defy precise diagnosis. Nonetheless, primary care meets a great majority of patient needs without referral.

4. It coordinates care. Primary care adopts mechanisms that facilitate the transfer of information about health needs and health care over time. Highly personalized solutions to patients' problems can be implemented when sustained relationships permit deeper knowledge and understanding of individuals' habits, preferences, and goals.

increasingly limited health care resources. Integrating genomic medicine into an engaged and resourced $\mathrm{PCMH}$, where personalized medicine is grounded in continuous healing relationships, ${ }^{19}$ could ensure its beneficial impact on health care.

In 2001-2002, Burke, Emery, and Hayflick challenged the primary care community to lead integration of genomics into practice through genetic literacy, collaboration with genetic counselors and genetic specialists, genetic risk assessment grounded in family history-taking, and

sive, coordinated) (Table 1) also address the complex challenges of delivering genomic medicine. ${ }^{6,7}$ Genomic medicine encompasses a wide range of testing applications, from prenatal and neonatal testing to germ line and tumor diagnostics, risk stratification, and pharmacogenomics, and the guidelines for testing and interpreting results include family history and collaboration with subspecialists.

Two IOM reports ${ }^{8,9}$ shed light on the quality chasm in the fragmented US health care system, and the Future of Family Medicine project ${ }^{10}$ called for primary care redesign, now known as the $\mathrm{PCMH}$. PCMH principles (eg, personal physician care, physician-directed teams, whole-person orientation, coordinated and integrated care, quality and safety, enhanced access, and payment for value) were adopted by many academic and professional organizations and employers to modernize frontline practice and achieve evidence-based care targets, which are well documented in other countries. ${ }^{11}$ The National Demonstration Project showed that implementation of the key PCMH features ${ }^{12,13}$ can improve quality and access to preventive health services. ${ }^{14,15}$ This national experience highlighted the need for external technical and systems support for practice transformation, health services research, and clinical implementation of PCMH features that work best for different strata of patient risk and health care utilization. ${ }^{12,16,17}$

There are opportunities within the national PCMH transformation movement (because of its successful experience with implementation, improving the Triple Aim of better health, better care, and lower costs, and utilizing team-based care coordination to improve patient outcomes) to generate value and synergy by fostering collaboration with genomic medicine implementation (Table 2).$^{18}$ Both efforts require practice redesign, better data, and decision support at a time of educational programs and decision support tools. ${ }^{20,21}$ Despite progress with disseminating core genomic concepts to primary care educators, ${ }^{22-24}$ implementation of clinical genomics into primary care-and clinical practice at large- - has been limited, primarily because, as Feero noted, "genomics remains a science of discovery rather than of clinical utility in most areas of medicine, despite enormous progress over the last decade," because a myriad of challenges must be overcome to integrate genomics in a complex, fragmented, health care system. ${ }^{18}$

Manolio and colleagues recently reported a consensus perspective from genomic medicine leaders affiliated with the National Human Genome Research Institute, which focused on designing a roadmap to guide clinical implementation across health systems (Table 2). ${ }^{18}$ Their report included systematic evidence reviews and protocols from successful projects and issued guidance for initiating pilot projects, evaluating outcomes, and disseminating findings to drive improvements. The National Demonstration Project overcame similar challenges by showcasing quality improvement efforts in primary care. For example, the Michigan Primary Care Transformation (MiPCT) project provided team-based care for hundreds of thousands of patients with chronic diseases. ${ }^{26,27}$ Another example is the University of California, Los Angeles/University of Southern California safety-net PCMH program, which demonstrated the benefits of care coordination on patient-reported coordination and access. ${ }^{28}$ Other programs in the Pacific Northwest have shown reduced health care utilization, emergency department visits, and costs, as well as improved quality, with PCMH transformation. ${ }^{29,30}$

Integrating Genomic Medicine and Primary Care Any vision for comprehensive primary care redesign should include a strategy for integrating genomic 
Table 2. Challenges in Implementation of Genomic Medicine in Primary Care and Potential Solutions

\section{Challenge \\ Limited evidence and conflicting interpreta- tion of benefit/value \\ Lack of institutional and clinician acceptance}

Limited access to genomic medicine expertise and testing

Lack of standards for genomic applications

EHR integration of genomic results and CDS

Follow-up of patients after genotyping

Outreach to at-risk family members

Consent

\section{Potential Solutions}

Generate evidence of clinical utility of genomic medicine interventions in PCMH settings

Tailor needs for evidence against potential benefits and harms

Convene expert panels to develop primary care guidelines based on best evidence

Engage community-based practices to assist in developing evidence

Determine process outcomes of incorporating genomic information into EHRs

Publicize success widely

Organize consortia to conduct practice-based research trials of genotype-derived therapy when appropriate Carry out RE-AIM framework evaluation at the level of the PCMH or PCMH network (Figure 1)

Establish institutional advisory committee(s) involving senior leadership and partner with early adopter programs to evaluate evidence, recommend and monitor implementation-at health care centers, community or regional levels-consistent with principles of primary care (Table 1) and community values

Engage early adopters and clinical champions in demonstration efforts

Conduct pilot projects in early adopter PCMHs to develop results sufficient for follow-on funding

Obtain transinstitutional commitment at highest levels involving all relevant departments and stakeholders

Utilize internal pilot funding to catalyze initiation

Build clinician acceptance of clinical genetics professionals by judiciously integrating genetic counselors and/or geneticists in nongenetics clinical services throughout primary institution/clinic and affiliated institutions

Bring fragmented expertise for advancing genomic medicine under 1 transdisciplinary PCMH program or network

Harness institutional (clinic or affiliated health care center) quality improvement processes to assess value

Use research-screening assays on site and confirm clinically actionable findings with rapid, cost-effective, CLIAcertified off-site testing if necessary

Establish or expand institutional CLIA-compliant genotyping to expand point-of-care testing, same-day service Choose platform to assay multiple important genotypes simultaneously, reliably, and inexpensively

Invest in new equipment and personnel to ensure research quality is at the same level as the clinical laboratory; this effort requires institutional investment

Work with genetic counselors in PCMH teams to establish protocols for process and parameters of data return Develop agreed-upon framework or standards for evaluation of genomic medicine applications

Develop standardized order sets and process modification

Develop standards for analytic validity of whole-genome and whole-exome sequencing sufficient for clinical interpretation of the variants found by these methods

Enable access to actionable genomic information in the EHR through development of user-friendly decision-support algorithms for primary care clinicians

Establish a "usability lab" to test genomic medicine applications in the EHR and assess CDS tools

Allocate genomic medicine institutional funding to develop education and outreach to disseminate best practices incorporating family history and genomic information

Redesign EHR to include section dedicated to containing all relevant genotype results for each patient

Develop and link actionable drug-gene pair decision to electronic pharmaceutical ordering software at point-of-care adoption of pharmacogenomic testing

Establish interdisciplinary workgroup with genomic medicine, chronic care, and EHR team to create secure tools for EHR-based genomic decision support

Establish ordering protocols to prompt appropriate referrals to genetic counselors based upon type and indication of genomic testing ordered

Shift from relying on primary care clinician direct contact to $\mathrm{PCMH} /$ genomic medicine teams with permission of clinician

Analyze and address reasons for refusal to complete confirmatory testing, such as lack of coverage for testing Clarify implications for family members and clinicians' responsibilities toward family members

Explore ways to improve information to at-risk families

Ensure that informed consents for implementation projects conducted as research studies include returning results to patients and entering results into EHR

Conformance with standard of care and specific consent may not be needed

Consider implementation projects that might not require consent, such as results in established clinical pathways (tumor mutations and germline polymorphisms affecting treatment decisions) in partnership with medical subspecialists who have content-specific expertise

Develop standards for informed consent for extensive genotyping or sequencing, including whole-genome sequencing, and obtain it prospectively and combine with protocols for testing indications

Ensure that availability of personnel to manage consent/counseling is not rate limiting in initial implementation

CDS = clinical decision support; CLIA = Clinical Laboratory Improvement Amendments; $E H R=$ electronic health record; $P C M H=$ patient-centered medical home RE-AIM = reach, effectiveness, adoption, implementation, and maintenance. 
Table 2. Challenges in Implementation of Genomic Medicine in Primary Care and Potential Solutions (continued)

\begin{tabular}{|c|c|}
\hline Challenge & Potential Solutions \\
\hline $\begin{array}{l}\text { Understanding by } \\
\text { patients, clinicians, } \\
\text { public }\end{array}$ & $\begin{array}{l}\text { Conduct focus groups of patients, clinicians, and ancillary personnel to identify specific educational needs } \\
\text { Conduct genetic and genomic medicine campaign for patients, clinicians, and ancillary personnel based on focus } \\
\text { group input } \\
\text { Survey retention of educational information by patients and clinicians and modify programs as needed } \\
\text { Conduct genotyping and/or sequencing and interpretation exercises with medical and other health profession } \\
\text { students } \\
\text { Provide specific health care clinician education on when to order tests, and how to interpret results, and how to act } \\
\text { on implications for family members and clinician's responsibilities } \\
\text { Introduce pharmacogenomic lectures into health professionals' training and continuing education } \\
\text { Provide clinical supervision to clinician trainees in use of pharmacogenomic testing, other genomic point-of-care } \\
\text { testing } \\
\text { Include American Board of Family Medicine (and other boards) maintenance of certification self-assessment modules } \\
\text { in genomic medicine } \\
\text { Development and dissemination of new educational objectives by the National Human Genome Research Institute } \\
\text { Inter-Society Coordinating Committee for Practitioner Education in Genomics }\end{array}$ \\
\hline $\begin{array}{l}\text { Lack of access to com- } \\
\text { parison "control" } \\
\text { sequence data and } \\
\text { banking resources }\end{array}$ & $\begin{array}{l}\text { Combine current PCMH's small patient collections of reference sequences and make available to all centers } \\
\text { Prioritize funding for costly and time-consuming storage of viable tissues/biospecimens for DNA analyses } \\
\text { Biobank tissues/biospecimens for confirmatory clinical sequencing with patient identifiers } \\
\text { High-level institutional or PCMH network commitment to combine and organize multiple biorepositories for effi- } \\
\text { ciency and ease of access while protecting patient privacy }\end{array}$ \\
\hline $\begin{array}{l}\text { Lack of research funding } \\
\text { and reimbursement }\end{array}$ & $\begin{array}{l}\text { Until evidence is established for making genomic testing a new standard of care, consider research funding for test- } \\
\text { ing in interim between discovery and adoption into PCMHs } \\
\text { Gradually change culture to convince health care community and patients of value of genomic medicine and need } \\
\text { for reimbursement } \\
\text { Demonstrate cost of testing is not prohibitive and savings impact can be substantial } \\
\text { Provide institutional back-up for reimbursement to avoid charges to patients } \\
\text { Anticipate rises in interpretive and delivery costs as technology cost drops and enthusiasm increases }\end{array}$ \\
\hline
\end{tabular}

CDS = clinical decision support; CLIA = Clinical Laboratory Improvement Amendments; EHR = electronic health record; PCMH = patient-centered medical home; RE-AIM = reach, effectiveness, adoption, implementation, and maintenance.

Note: Challenges to clinical implementation identified by a National Human Genome Research Institute Genomic Medicine Colloquium representing 20 health care organizations and working groups in June $2011 .{ }^{16}$ Table adapted to the goal of integration of genomic medicine clinical implementation with primary care redesign

medicine with primary care in academic and nonacademic settings and engage genomic medicine leaders in developing a clinical implementation strategy. Figure 1 illustrates a proposed roadmap for integration that includes 3 components: (1) evidence analysis and gap filling, (2) clinical implementation science, and (3) clinical implementation within a continuous improvement loop whereby clinical observations inform translational science and dissemination.

\section{EVIDENCE ANALYSIS AND GAP FILLING}

The scope of genomic medicine ranges from diagnostic testing for rare diseases to cancer syndromes, longitudinal testing and surveillance of probands and family members, prenatal carrier screening, disease risk prediction, and pharmacogenomics. Genomic research informs drug discovery and development, resulting in potentially more effective therapies for such rare diseases as cystic fibrosis and such common diseases as advanced lung cancer. Pharmacogenomics is not yet a routine part of practice in primary care, yet more than
150 Food and Drug Administration drug labels contain guidance on pharmacogenomic testing to inform drug selection, dosing, or toxicity prevention. ${ }^{31}$

Evidentiary quality of genomic testing relies on analytic validity, clinical validity, and clinical utility. ${ }^{32}$ Analytic validity describes the accuracy of a genomic test for its intended measure. Clinical validity assesses whether a relationship exists between a genomic test and a clinical phenotype. Clinical utility is the degree to which a genomic result informs a change in management that improves clinical outcomes.

Because of the underdevelopment of clinical utility data, evidence-based guidelines exist for only a small subset of genomic tests (mostly diagnostic in nature). Accordingly, a systematic, multidisciplinary approach to addressing evidence gaps is needed ${ }^{33}$ to align evidence with established validity, safety, and effectiveness thresholds. ${ }^{34}$ Few studies provide sufficient clinical utility, however. For example, most genome-wide or wholeexome analyses are conducted within cross-sectional or cohort studies, and examples with minority populations are scarce. Other shortcomings include widespread 
publication bias, small sample sizes, low power, and overestimated effects. Information is limited for implementing genomic medicine into ambulatory care practices that comprise multidisciplinary teams.

These issues underlie some of the barriers to widespread clinical implementation. ${ }^{35}$ As gaps in scientific discovery and decision support are filled and aligned with public health priorities, the utility and value to health care systems will drive investment in clinical implementation beyond academic medical centers and into PCMHs.

\section{CLINICAL IMPLEMENTATION SCIENCE}

The reach, effectiveness, adoption, implementation, and maintenance (RE-AIM) framework was introduced $^{36}$ to address the need to transfer knowledge from efficacy research conducted under optimal conditions into complex, real-world systems. RE-AIM follows a logical sequence to evaluate and promote clinical implementation.

The reach of innovation (eg, genomic testing) refers to number, proportion, and representativeness of the clinicians willing to use the innovation. ${ }^{36}$ Before introducing genomic testing or clinical initiatives, pre- implementation surveys can gauge interest and demand so implementation efforts can be focused on early adopters.

Effectiveness refers to improved patient outcomes within a clinical population. ${ }^{36}$ Once genetic testing is implemented in primary care settings, it is important that improvements in behavioral and clinical outcomes validate its impact. Moreover, evidence to guide whom to test and how to interpret the results should be used to develop clinical decision support tools within electronic health records (EHRs). A recent exploratory study at Stanford University underscores this point. Dewey et al conducted whole-genome sequencing in adults using 2 separate sequencing platforms. They found that depending on the sequencing platform, $10 \%$ to $19 \%$ of inherited disease genes were not covered to accepted standards for single nucleotide polymorphism (SNP) discovery, ${ }^{35}$ even though concordance was high for previously described SNP variants (99\% to $100 \%)$, it was low for other variants (53\% to 59\%). Most (69\%) of variants previously classified as causing disease were reclassified as variants of uncertain significance after expert review. Although whole-genome sequencing is not recommended for routine screening in primary care, and clinical-grade genomic tests are available in

Figure 1. Schematic roadmap for clinical implementation of genomic medicine in primary care.

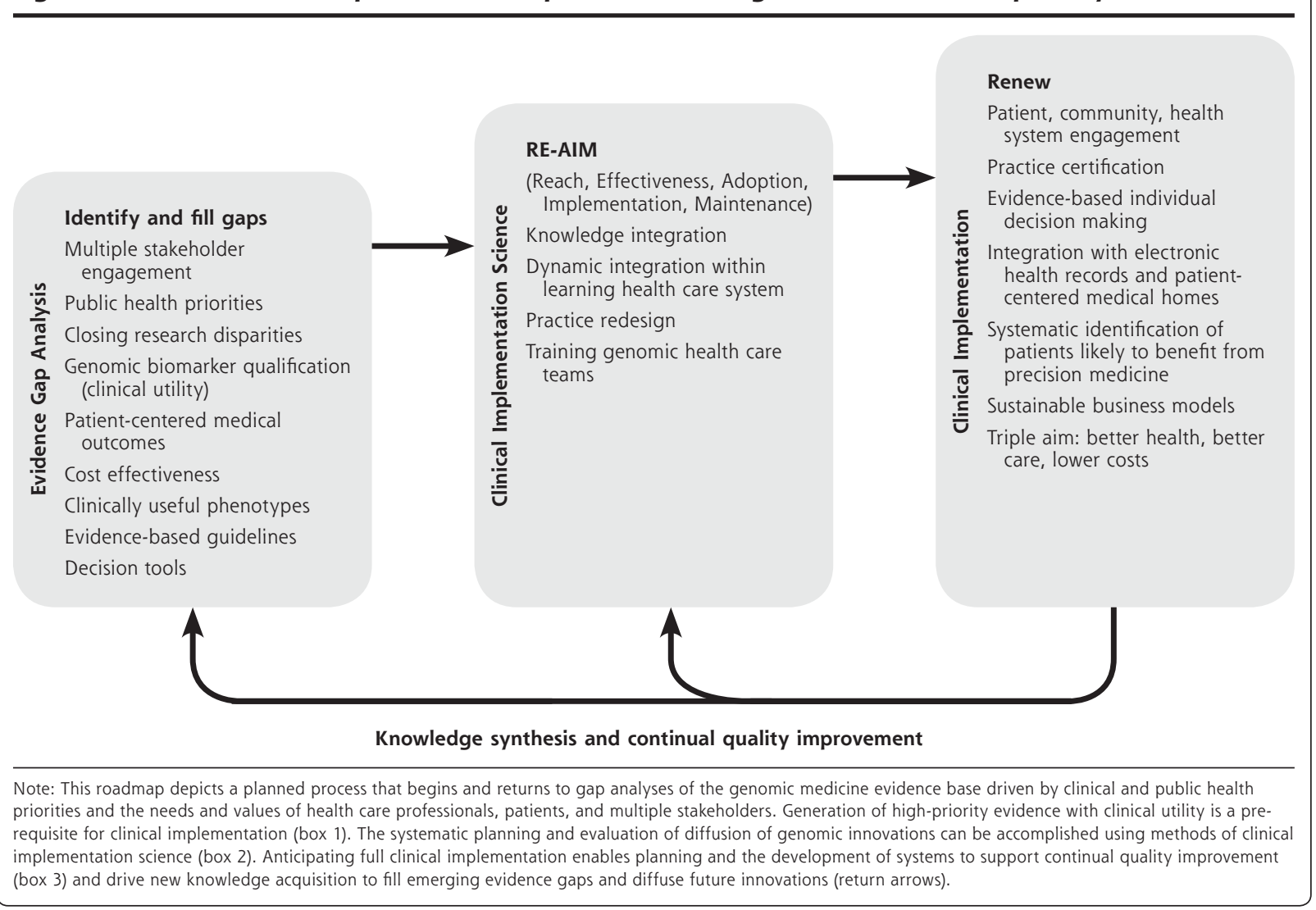


multiple clinical centers, ${ }^{37}$ the study by Dewey et $\mathrm{al}^{35}$ showed that even though whole genome sequencing can benefit certain individuals, technical issues, such as incomplete coverage of inherited disease genes, limited reproducibility of genetic variants with the highest clinical benefits, and considerable time for expert review per patient, will need to be addressed when determining its role in clinical practice.

Evidence-based clinical criteria for screening and interpreting genomic variants and prescribing could be annotated within EHRs by using such Web-based tools as UpToDate (Wolters Kluwer) and DynaMed (EBSCO). Examples of genomic medicine guidelines include the Evaluation of Genomic Applications in Practice and Prevention (EGAPP) guidelines for genomic testing (http://www.egappreviews.org), the National Comprehensive Cancer Network (NCCN) guidelines for detection, prevention, and risk reduction of cancer (http://www.nccn.org), and the Clinical Pharmacogenomics Implementation Consortium (CPIC) guidelines for drug selection and dosing (http://www. pharmgkb.org/page/cpic). Such resources could enable systematic, evidence-based, point-of-care decision support to help determine which patients would benefit from genomic predisposition screening or detection of drug-gene interactions. Combined with validated family history screening algorithms, ${ }^{38}$ health behaviors, and other risk factors, patients could be stratified by risk for prevention interventions. ${ }^{18,39-41}$

Adoption includes number, proportion, and representativeness of clinicians and other individuals interested in genomic medicine implementation. ${ }^{36}$ Multiple stakeholders must be engaged to enable adoption. An adoption assessment estimates the program's potential value and patient demand, moreover, awareness of genomic medicine-including facilitators and barriers-further enables success.

Implementation refers to the extent to which a program is delivered as intended in a real-world setting. ${ }^{36}$ Implementation can also be thought of as interacting with efficacy to determine effectiveness (efficacy $\times$ implementation $=$ effectiveness). The National Institutes of Health (NIH)-funded Electronic Medical Records and Genomics (eMERGE) Consortium proposed a definition of successful implementation as (1) generation of new knowledge, (2) dissemination of knowledge, (3) translating personalized medicine into clinical practice, and (4) transforming health care by empowering patients with data. ${ }^{42}$

Maintenance is how a program is sustained over time. ${ }^{34}$ Maintenance includes program-level measures of institutionalization and is based on the extent to which genomic medicine becomes part of routine primary care and the norms of practice.
Extending the RE-AIM framework beyond academic medical centers to wide-scale PCMH projects helps identify best practices and expand the reach of primary care-based genomic medicine. This step is critical to creating the institutional evidence base for clinical implementation in community-based primary care practices. The evaluation of maintenance of genomic-based practices-once implemented-should be a dynamic process that informs research needs and guides future efforts.

\section{Clinical Implementation and Evaluation}

Beyond clinical implementation science into a future health care system informed by the RE-AIM framework, principles of continuing quality improvement would enable incorporation of advances in genomicsbased discoveries. Clinical and patient safety outcomes could be monitored and measured within the framework to encourage value-driven innovation. Using standardized EHR terminology to link phenotypes with clinical outcomes could validate the effectiveness of interventions after health system adoption. Required would be continuous reevaluation of technologies to facilitate identifying errors in medical records and mistakes in medical decisions informed by genomic testing. Houwink and colleagues proposed a roadmap for stepwise integration of family history data with primary care and genomic services based upon the Dutch research experience, which provides a useful illustration for primary practices and health care centers undergoing clinical implementation. ${ }^{43}$ Emery and colleagues, in the United Kingdom and Australia, have already shown that combining primary care education, partnership models, and systematic integration of the family history within EHRs is effective in improving hereditary cancer screening. ${ }^{21,38}$ Finally, multiple US health care systems-including the Cleveland Clinic, Duke University Medical Center, Intermountain Healthcare, Vanderbilt University Medical Center, and St. Jude Children's Research Hospital — have successfully integrated family history and genomic medicine within EHRs. ${ }^{18}$

Given the complexity and heterogeneity of care delivery across primary care practice settings, genomic medicine integration must align with translational research to optimize use and cost of personalized health care and continue to engage and educate the primary care workforce. Because genomic discovery often outpaces clinical guideline development, educational efforts have shifted to emphasize practical, electronic, clinical decision support tools (including point-of-care educational materials) that capitalize on multi-institutional collaboration and dissemination of best practices. ${ }^{18}$

If the roadmap to genomic medicine integration into PCMHs (Figure 1) is successfully followed in 
partnership with primary care and genomics professionals, the need for evidence-gap analyses and gap filling should diminish (box 1). Primary care physicians will be more informed and engaged, genomic medicine will be systematically adopted by multidisciplinary teams, and the health outcomes can be promoted and measured with the RE-AIM framework (box 2). Once realized, the future of genomic medicine will focus on continual quality improvement and promotion of population health (box 3 ).

Re-engineering the health care system to integrate genomic medicine is an ambitious task. It would be possible to instill continual quality improvement and a culture of renewal into this system by incorporating iterative processes that ask what can be done better, what improves efficiency, and what adds value to patient-centered outcomes. In this way, the system can adapt as knowledge accumulates ahead of adoption.

Integration of genomic medicine into a primary care renewal process offers the potential to catalyze clinical implementation and contribute to improved patientcentered outcomes. Making personalized health care even more personal, however, requires reaiming translational pipelines toward greater healing relationships in a continuously improving PCMH. Doing so could enable realization of the primary care-genomics partnership models envisaged by leaders in both fields. ${ }^{20,21,44}$

To read or post commentaries in response to this article, see it online at http://www.annfammed.org/content/13/4/373.

Key words: genomics; primary health care; personalized medicine; clinical implementation science; precision medicine

Submitted June 3, 2014; submitted, revised, December 29, 2014; accepted January 21, 2015.

Funding support: Drs David and Johnson were fellows with the Institute of Medicine Roundtable on Translating Genomics-based Research for Health

Disclaimer: The views expressed in this article are those of the individual authors and do not necessarily represent the views of their affiliated organizations, institutions, government agencies, the Institute of Medicine, or the United States government.

Acknowledgments: The authors thank the members of the IOM Roundtable on Translating Genomic-Based Research for Health, particularly Wylie Burke, MD, PhD, from the University of Washington, Seattle, Washington, and Russell Glasgow, MS, PhD, from the University of Colorado, Denver, Colorado, for their thoughtful reviews and insights.

\section{References}

1. Peabody FW. The care of the patient. JAMA. 1927;88(12):877-882.

2. Centers for Disease Control and Prevention. National Ambulatory Medical Care Survey: 2010 Summary Tables. http://www.cdc.gov/ nchs/data/ahcd/namcs_summary/2010_namcs_web_tables.pdf. Published 2010
3. Green LA, Fryer GE Jr, Yawn BP, Lanier D, Dovey SM. The ecology of medical care revisited. N Engl J Med. 2001;344(26):2021-2025.

4. Collins F. Has the revolution arrived? Nature. 2010;464(7289):674-675.

5. Institute of Medicine. Primary Care: America's Health in a New Era. Washington, DC: The National Academies Press; 1996.

6. Starfield B, Horder J. Interpersonal continuity: old and new perspectives. Br J Gen Pract. 2007;57(540):527-529.

7. Institute of Medicine. Primary Care and Public Health: Exploring Integration to Improve Population Health. Washington, DC: The National Academies Press; 2013.

8. Institute of Medicine. To Err is Human: Building a Safer Health System. Washington, DC: The National Academies Press; 2000.

9. Institute of Medicine. Crossing the Quality Chasm: A New Health System for the 21st Century. Washington, DC: The National Academies Press; 2001.

10. Martin JC, Avant RF, Bowman MA, et al. The future of family medicine: a collaborative project of the family medicine community. Ann Fam Med. 2004(3(Suppl 1):S2-S99

11. Starfield B, Shi L, Macinko J. Contribution of primary care to health systems and health. Milbank Q. 2005;83(3):457-502.

12. Crabtree BF, Nutting PA, Miller WL, Stange KC, Stewart EE, Jaén CR. Summary of the National Demonstration Project and recommendations for the patient-centered medical home. Ann Fam Med. 2010;8(Suppl_1):S80-S90;S92.

13. Baird $M$, Blount $A$, Brungardt $S$, et al. Joint principles: integrating behavioral health care into the patient-centered medical home. Ann Fam Med. 2014;12(2):183-185.

14. Jackson GL, Powers BJ, Chatterjee R, et al. Improving patient care. The patient centered medical home. A systematic review. Ann Intern Med. 2013;158(3):169-178.

15. Jaén $C R$, Ferrer RL, Miller WL, et al. Patient outcomes at 26 months in the patient-centered medical home National Demonstration Project. Ann Fam Med. 2010;8(Suppl_1):S57-S67;S92.

16. Nutting PA, Miller WL, Crabtree BF, Jaén $C R$, Stewart EE, Stange KC. Initial lessons from the first national demonstration project on practice transformation to a patient-centered medical home. Ann Fam Med. 2009;7(3):254-260.

17. Schwenk TL. The patient-centered medical home: one size does not fit all. JAMA. 2014;311(8):802-803.

18. Manolio TA, Chisholm RL, Ozenberger B, et al. Implementing genomic medicine in the clinic: the future is here. Genet Med. 2013;15(4):258-267.

19. Starfield B. The future of primary care: refocusing the system. N Engl J Med. 2008;359(20):2087-2091.

20. Burke W, Emery J. Genetics education for primary-care providers. Nat Rev Genet. 2002;3(7):561-566.

21. Emery J, Hayflick $S$. The challenge of integrating genetic medicine into primary care. BMJ. 2001;322(7293):1027-1030.

22. Laberge AM, Fryer-Edwards K, Kyler P, Lloyd-Puryear MA, Burke W. Long-term outcomes of the "Genetics in Primary Care" faculty development initiative. Fam Med. 2009;41(4):266-270.

23. Saul RA. Genetic and genomic literacy in pediatric primary care. Pediatrics. 2013;132(Suppl_3):S198-S202.

24. Burke S, Martyn M, Stone A, Bennett C, Thomas H, Farndon P. Developing a curriculum statement based on clinical practice: genetics in primary care. Br J Gen Pract. 2009;59(559):99-103.

25. Feero WG. Genomics in medicine: maturation, but not maturity. JAMA. 2013;309(14):1522-1524.

26. Harcus A. MiPCT: the nation's largest patient-centered medical home project. Mich Med. 2011;110(5):10-11.

27. Paustian ML, Alexander JA, El Reda DK, Wise CG, Green LA, Fetters MD. Partial and incremental PCMH practice transformation: implications for quality and costs. Health Serv Res. 2014;49(1):52-74. 
28. Hochman ME, Asch S, Jibilian A, et al. Patient-centered medical home intervention at an internal medicine resident safety-net clinic JAMA Intern Med. 2013;173(18):1694-1701.

29. Driscoll DL, Hiratsuka V, Johnston JM, et al. Process and outcomes of patient-centered medical care with Alaska Native people at Southcentral Foundation. Ann Fam Med. 2013;11(Suppl 1):S41-S49.

30. Reid RJ, Coleman K, Johnson EA, et al. The Group Health medical home at year two: cost savings, higher patient satisfaction, and less burnout for providers. Health Aff (Millwood). 2010;29(5):835-843.

31. US Food and Drug Administration. Table of Pharmacogenomic Biomarkers in Drug Labeling. http://www.fda.gov/Drugs/ScienceResearch/ResearchAreas/Pharmacogenetics/ucm083378.htm. Updated Aug 18, 2014. Accessed Apr 26, 2015.

32. Institute of Medicine. Genome-based Diagnostics: Demonstrating Clinical Utility in Oncology-Workshop Summary. Washington, DC: The National Academies Press; 2013.

33. Institute of Medicine. Refining Processes for the Co-development of Genome-based Therapeutics and Companion Diagnostics-Workshop Summary. Washington, DC: The National Academies Press; 2014.

34. Feero WG, Wicklund C, Veenstra DL. The economics of genomic medicine: insights from the IOM Roundtable on Translating Genomic-Based Research for Health. JAMA. 2013;309(12):1235-1236.

35. Dewey FE, Grove ME, Pan C, et al. Clinical interpretation and implications of whole-genome sequencing. JAMA. 2014;311(10): 1035-1045.

36. Glasgow RE, Vogt TM, Boles SM. Evaluating the public health impact of health promotion interventions: the RE-AIM framework. Am J Public Health. 1999;89(9):1322-1327.
37. Grody WW, Vilain E, Nelson SF. Interpreting whole-genome sequencing. JAMA. 2014;312(3):296.

38. Emery JD, Reid G, Prevost AT, Ravine D, Walter FM. Development and validation of a family history screening questionnaire in Australian primary care. Ann Fam Med. 2014;12(3):241-249.

39. Qureshi N, Armstrong S, Dhiman P, et al; ADDFAM (Added Value of Family History in CVD Risk Assessment) Study Group. Effect of adding systematic family history enquiry to cardiovascular disease risk assessment in primary care: a matched-pair, cluster randomized trial. Ann Intern Med. 2012;156(4):253-262.

40. Qureshi N, Carroll JC, Wilson B, et al. The current state of cancer family history collection tools in primary care: a systematic review. Genet Med. 2009;11(7):495-506.

41. Rubinstein WS, Acheson LS, O'Neill SM, et al; Family Healthware Impact Trial (FHITr) Group. Clinical utility of family history for cancer screening and referral in primary care: a report from the Family Healthware Impact Trial. Genet Med. 2011;13(11):956-965.

42. Kannry JL, Williams MS. Integration of genomics into the electronic health record: mapping terra incognita. Genet Med. 2013; 15(10):757-760.

43. Houwink EJ, Sollie AW, Numans ME, Cornel MC. Proposed roadmap to stepwise integration of genetics in family medicine and clinical research. Clin Transl Med. 2013;2(1):5.

44. Collins FS, Green ED, Guttmacher AE, Guyer MS; US National Human Genome Research Institute. A vision for the future of genomics research. Nature. 2003;422(6934):835-847.

\section{family medicine}

\section{Now Available}

You've been hearing about the Family Medicine Residency Curriculum Resource for nearly 3 years. It's finally here, with more than 70 peer-reviewed competency based curricula, organized by post-graduate year. Each curriculum includes a case-based presentation, facilitators' guide and quiz. New curricula will be added almost weekly throughout 2015.

Learn more and purchase a subscription for your program at www.fammedrcr.org. 